\section{SASOP treatment guidelines for psychiatric disorders: Eminence or evidence based?}

To the Editor: In August 2013, the South African (SA) Society of Psychiatrists (SASOP) published their treatment guidelines for psychiatric disorders. ${ }^{[1]}$ We commend the authors and editors for completing such a huge undertaking. In the spirit of ongoing peer review, we would like to point out a few issues that may warrant consideration for future guideline updates.

Although the authors seem to be critical of 'evidence-based medicine' (EBM) and mention that different interpretations of the evidence base in psychiatry are often selectively applied to support a particular point of view, they seem nevertheless to advocate that guidelines be backed up by evidence. However, the various guidelines fail to use a standardised and consistent methodology for appraising the evidence. This is despite the fact that such EBM tools exist, such as the Grading of Recommendations Assessment, Development and Evaluation (GRADE) system, ${ }^{[2]}$ the Appraisal of Guidelines for Research and Evaluation (AGREE-II) tool ${ }^{[3]}$ and the World Health Organization's guideline development instrument. ${ }^{[4]}$

There seems to be an absence of methodological input, with each guideline written by a single content expert only. In our opinion, the scope and purpose of each guideline lacks detail, particularly with regard to the patient population, particular treatment comparisons and specific patient-relevant outcomes. In addition, there is no clearly defined systematic search strategy for the literature and we are left to the mercy of 'file drawer' bias, with many statements made in the guidelines lacking appropriate referencing. There appears to be no consistent methodology to appraise the quality of the evidence and its strengths and weaknesses, and no explicit and transparent framework for how clinical recommendations are reached. Whereas there have been attempts by some authors to grade evidence in some way, these attempts have limitations that are made apparent by placing emphasis on meta-analysis, which seems to be conflated with systematic reviews. Furthermore, low-quality observational research is often used to support specific recommendations.

Whereas reinventing the wheel is clearly inappropriate, and many guidelines already exist that have some external validity applicable to the SA context, such guidelines also need to be approached with caution because the barriers and facilitators to implementation in the SA (private sector) context of certain treatments remain unique. There is little discussion of how certain psychological, psychosocial and pharmacological interventions, used in other international settings, could be generalised to the SA setting. Furthermore, the extent of stakeholder involvement, both from a patient and provider perspective, is not clearly conveyed, particularly with regard to different values and level importance ascribed to certain outcomes.

One potential dilemma is the dearth of necessary expertise to embark on guidelines of such a magnitude. Despite these problems, it would be good to see a more consistent and developed methodology for future guidelines, whereby each guideline panel for different disorders consists of more than one member, and includes content and method experts, and where a transparent and explicit framework is followed in order to appraise the evidence and to reach decisions regarding treatment recommendations that strike the best balance between benefit and risk of harm. Furthermore, it would be encouraging to see a variety of potential stakeholders such as the Medical Research Council, Department of Health and university departments collaborating to fund and support guideline development, regardless of whether they are intended for use in the private or public sector.

\section{S Baumann, J Benson-Martin, Q Cossie, K Gilfillan, N Horn, $S$ Kaliski, P Milligan, $H$ Temmingh, T Timmermans, P Williams-Ashman}

Department of Psychiatry and Mental Health, Valkenberg Hospital, University of Cape Town, South Africa

\section{References \\ 1. Emsley R, Seedat S (eds). The South African Society of Psychiatrists (SASOP) Treatment Guidelines for Psychiatric Disorders. South African Journal of Psychiatry 2013;19(3):128-199. [http://dx.doi org/10.7196/SAJP.474] \\ 2. Guyatt G, Oxman AD, Akl EA, et al. GRADE guidelines: 1. Introduction - GRADE evidence profiles and summary of findings tables. J Clin Epidemiol 2011;64(4):383-394. [http://dx.doi. org/10.1016/j.jclinepi.2010.04.026] \\ 3. Brouwers MC, Kho ME, Browman GP, et al. AGREE II: Advancing guideline development, reporting and evaluation in healthcare. CMAJ 2010;182(18):e839-842. [http://dx.doi.org/10.1503/cmaj.090449] \\ 4. World Health Organization. WHO Handbook for Guideline Development. Geneva: World Health Organization Press, 2012. http://apps.who.int/iris/bitstream/10665/75146/1/9789241548441_eng. pdf (accessed 16 October 2012).}

Response invited by the Editor: In responding to this letter I believe that it is essential to begin with the history of the guidelines. Already ten years ago, the then president of SASOP, Dr Eugene Allers, advocated for the creation of South African treatment guidelines in psychiatry. These guidelines had become critically important for two reasons: Firstly, algorithms for psychiatric treatment did not exist at that stage, and often State psychiatry would find itself in a very limited and often non-evidence-based vice grip regarding the availability of treatment methods. Secondly, private psychiatrists were progressively exposed to so-called 'medication algorithms' created by medical schemes and applied indiscriminately, often based on financial considerations rather than evidence-based medicine. It had therefore become essential to create some form of guideline to formulate the position of psychiatrists in South Africa regarding treatment of commonly occurring psychiatric disorders.

The process was time consuming. Prof. Robin Emsley and Prof. Soraya Seedat took over the immense task of coordinating the writing of the guidelines. It was also very difficult to find people committed enough to compile these guidelines. In the end, the guidelines were a product of many hours of hard work, done by single authors, notwithstanding the meticulous peer-review process to which each chapter was subjected.

When compiling these guidelines, the compilers and reviewers were requested to refer to existing international treatment guidelines such as the International Society for Bipolar Disorders guidelines, the Canadian Network for Mood and Anxiety Treatments (CANMAT) 


\section{LETTER}

guidelines and many other excellent guidelines existing in the literature. These formed the basis for an attempt at creating an initial document in this very difficult field.

We agree with the authors that many excellent international guidelines exist. However, uncritical application to our local situation would be unwise. The letter also rightly mentions that there is a lack of critical mass in terms of expertise to embark on guidelines of such magnitude, and mentions that there are several resources that could be used to improve these guidelines, for example the Medical Research Council, the Department of Health and university departments collaborating in terms of funding guideline development, whether intended for the private or public sector. I am, however, sceptical about the willingness to undertake, and availability of resources for, such a project, particularly from the Department of Health.

One should certainly take these criticisms seriously, but one should also acknowledge that this was a first attempt at creating something which, up until its publication, had not existed in contemporary South African psychiatry. Certainly the compilation of these guidelines, to a large extent, was grounded in a balance between 'ability' and 'willingness', rather than 'eminence. There is, furthermore, always the issue that when work gets done, only a few stalwarts usually 'rise to the occasion'. The guidelines were, as previously stated, intended to oppose the far worse and uncritically compiled treatment guidelines that had been created by many manage care organisations, leading to an infringement of patient rights and often denial of essential treatment.

It is heartening to note that ten eminent academic psychiatrists from the University of Cape Town have clearly taken this issue seriously and propose an excellent methodology, including within their critical and constructive letter a suggestion to proceed forward. I would respectfully suggest that this letter and my response be referred to the Board of Directors of SASOP for consideration that these ten psychiatrists be tasked with the substantial and immediate review of the treatment guidelines. It is often said that on the very day of publication of guidelines, they are already out of date in some respects. It would therefore be a process that could be started immediately, and I would suggest that the lead author, Dr S Baumann, be approached to coordinate such a task. I certainly agree with a broader base of literature review and compilation of guidelines, and I am personally looking forward to this essential product being improved and shaped according to the excellent suggestions contained in this letter to the editor.

\section{Franco Colin}

Department of Psychiatry, University of Pretoria

Private practice, Pretoria

S Afr J Psych 2014;20(2):63-64. DOI:10.7196/SAJP.529 